\title{
Antíteses sobre o mundo rural brasileiro: por uma visão para o futuro
}

\section{Antitheses of the Brazilian countryside: for a future vision}

Gabriel da Silva Medina - Professor da Universidade Federal de Goiás; pós-doutor em Políticas Ambientais (Imperial College London); doutor em Ciências Naturais (Universidade de Freiburg); mestre em Agriculturas Familiares e Desenvolvimento Sustentável (Universidade Federal do Pará). E-mail: gabriel.silva.medina@gmail.com

\section{Resumo}

Avanços importantes na compreensão do mundo rural brasileiro foram feitos a partir de teses sobre a formação de um novo padrão agrícola e agrário com base na expansão do agronegócio. Pesquisas recentes revelam a necessidade de revisitar essas teses a partir das seguintes antíteses: 1) o aumento da produção nas fronteiras agrícolas ainda é mais influenciado pela tradicional expansão da área cultivada, 2) a expansão para novas fronteiras gera altos custos socioambientais, 3 ) a aposta na liberalização dos mercados ignora políticas protecionistas de outros países, 4) há grande diversidade de agricultores com pequena produção, mas com potencial de desenvolvimento. Como síntese, o futuro do mundo rural brasileiro passa pela integração vertical do capital nacional ao longo da cadeia produtiva, superando a visão atual restrita à expansão para novas fronteiras agrícolas, com o Estado apoiando estratégias de longo prazo dos segmentos empresarial e familiar.

\begin{abstract}
The emergence of a new Brazilian agricultural and agrarian pattern underpinned by agribusiness expansion based on a small number of highly productive farmers is supported by different scientific theses. However, recent research results suggestthe need to revisit to these theses based on the following antitheses: 1. Growing production in agricultural frontiers is mainly due to the traditional increase of croplands; 2 . Agribusiness expansion into new agricultural frontiershave high social and environmental impacts; 3. Privet sector'semphasis on market liberalization ignores the protectionist policies adopted by other countries; 4 . There is great diversity of farmers with small production butwith development potential. Summing up, the future of Brazilian agribusiness depends on increasing the share of national capital for these businesses, while balancing the current strategy of expansion into new agricultural frontiers, with an important role played by the State in supporting both large and family-based farmers.
\end{abstract}

\section{Keywords}

Rural Development. Agribusiness. Family

Farming. Agricultural Policy. Strategy.
Desenvolvimento rural. Agronegócio. Agricultura familiar. Política agrícola. Estratégia. 


\section{INTRODUÇÃO}

Pesquisadores têm conseguido avanços importantes na compreensão das principais dinâmicas em curso no Brasil rural (GRAZIANO DA SILVA, 2013; SCHNEIDER, 2010; VEIGA, 2001). Um esforço adicional foi feito com a publicação de teses sobre o mundo rural que apontam para a formação de um novo padrão agrário e agrícola brasileiro com base na expansão do agronegócio privado feito por um número reduzido de agricultores altamente produtivos (BUAINAIN et al., 2013, 2014). Essencialmente as teses defendem o sucesso do agronegócio nacional exportador em contraste com as dificuldades enfrentadas pela maior parte das propriedades rurais que são consideradas redundantes (ALVES; ROCHA, 2010; BUAINAIN et al., 2013).

Estudos recentes, no entanto, têm mostrado a necessidade de dois passos adicionais na sistematização do conhecimento sobre o tema: 1) a revisitação a alguns dos elementos estruturantes das principais teses sobre as dinâmicas em curso, relativizando suas implicações (GUANZIROLI; DI SABBATO, 2014) e, 2) a necessidade de ir além da constatação da situação atual, elaborando uma visão que aponte para o futuro do desenvolvimento rural brasileiro (MEDINA, 2018).

Este artigo busca contribuir para a construção do conhecimento científico sobre o mundo rural brasileiro, apontando para uma visão de futuro. Especificamente, busca-se: 1. Sistematizar as antíteses sobre o mundo rural brasileiro a partir do conhecimento existente; e 2. Apontar para possibilidades de síntese em favor de uma visão sobrecaminhos para o desenvolvimento futuro. O artigo está estruturado em torno de quatro antíteses que contextualizam e relativizam as teses existentes. As antíteses são seguidas de um esforço inicial de síntese que indica alternativas de desenvolvimento.

\section{METODOLOGIA}

Este artigo foi elaborado a partir do método dialético, que parte de diferentes pontos de vista sobre um mesmo assunto e busca estabelecer a verdade a partir de discussões racionais amparadas em argumentos fundamentados. Os elementos do método dialético são a tese, a antítese e a síntese. A tese é uma afirmação ou situação inicialmente dada. A antítese é uma oposição à tese ou sua contextualização e relativização. Do contraste entre tese e antítese surge a possibilidade de síntese, que é uma situação nova que carrega dentro de si elementos resultantes desse contraste. 
Neste artigo, partiu-se de algumas das teses apresentadas por Buainain et al. (2013) sobre o mundo rural brasileiro no artigo "Sete teses sobre o mundo rural brasileiro". À luz de resultados de pesquisas recentes, foram sistematizadas quatro antíteses que permitiram relativizar alguns elementos estruturantes das teses iniciais. Um esforço final, embora ainda de caráter preliminar, foi feito como tentativa de síntese, que aponta para uma visão sobre as possibilidades de desenvolvimento futuro do mundo rural brasileiro. $\mathrm{O}$ trabalho foi elaborado com base na revisão da literatura recente sobre o assunto e na sistematização dos principais resultados de pesquisa existentes relacionados às teses iniciais.

\section{RESULTADOS}

2.1 O AUMENTO DA PRODUÇÃO NAS FRONTEIRAS AGRÍCOLAS AINDA É MAIS INFLUENCIADO PELA EXPANSÃO DA ÁREA CULTIVADA, QUE CONCENTRA A PARTICIPAÇÃO DO CAPITAL NACIONAL, DO QUE POR UM NOVO PADRÃO AGRÍCOLA

A primeira tese sugere que o Brasil estaria em uma nova fase de seu desenvolvimento agrícola e agrário caracterizada pela prevalência do capital como motor do agronegócio moderno. A dominação do capital, rebaixando o peso do fator terra, e o papel central das inovações como mecanismos principais de crescimento da produção agropecuária seriam evidenciados pela evolução da produtividade total de fatores (PTF) (BUAINAIN et al., 2013). Este argumento está baseado em um estudo que revela que, enquanto a produção da agropecuária cresceu 243\% entre 1970 e 2006, o uso de insumos cresceu apenas 53\% (GASQUES, 2010), o que indicaria que o crescimento da agricultura brasileira tem se dado principalmente com base na produtividade (BUAINAIN et al., 2013).

No entanto, um estudo posterior realizado para o bioma Cerrado como um todo revela que, dos $192 \%$ de aumento de produção observados no período de 1985 a 2006, 16\% podem ser atribuídos ao aumento da produtividade dos fatores enquanto que os restantes $176 \%$ se deram pelo uso adicional de recursos como terra, trabalho, materiais e capital (RADA, 2013). Os resultados revelam uma diferença substancial de produtividade entre os produtores mais eficientes e a média. O estudo aponta que a generalização das formas mais eficientes de produção vai ser cara em termos de insumos tais como fertilizantes e pesticidas, o que torna o seu sucesso dependente dos preços reais desses insumos (RADA, 2013).

A diferença nos resultados entre os dois estudos pode ser atribuída ao diferente recorte geográfico, ao período analisado e à metodologia adotada para 
o cálculo da PTF. Gasques (2010) analisou dados nacionais que são influenciados por estados em que a ocupação do território é mais antiga, como Minas Gerais, Bahia, Goiás e Paraná, e onde o aumento recente da produção é resultado principalmente do acúmulo de capital e do avanço tecnológico (GASQUES et al., 2014). Adicionalmente, a metodologia usada para os cálculos de PTF revela que o produto considerado é o resultado da agregação de lavouras temporárias, lavouras permanentes, produção animal e pecuária (GASQUES; BACCHI; BASTOS, 2018). Dessa forma, considera-se que parte importante do aumento da produtividade total pode ter se dado pela substituição de atividades menos produtivas (como pastagens para a criação extensiva de gado) por atividades mais produtivas (como soja). De fato, de 1975 a 2016, as áreas de pastagem no país foram reduzidas de 165 milhões para 145 milhões de hectares, enquanto as lavouras temporárias expandiram de 36,8 milhões para 69,5 milhões de hectares (GASQUES; BACCHI; BASTOS, 2018).

No caso do Cerrado, estudado por Rada (2013), a incorporação de novas áreas teve maior influência no aumento da produção do que o avanço tecnológico. Dessa forma, pelo menos nas novas fronteiras agrícolas (como é o caso de grande parte do Cerrado), o aumento da produção ainda é mais influenciado pela tradicional expansão da área cultivada do que por uma nova fase do desenvolvimento agrícola e agrário com alta produtividade. Outro estudo recente mostra que o aumento da produção de commodities no Brasil está relacionado tanto com o efeito área (aumento da área colhida) quanto com o rendimento (produtividade), o que evidencia um processo de expansão de terras disponíveis e, concomitantemente, a melhoria de sua produtividade (PEROBELLI et al., 2016).

Acerca da participação do capital no agronegócio feito no Brasil e suas implicações para o futuro do setor, o capital nacional está concentrado em insumos básicos como terra e mão de obra enquanto que a tecnologia e a governança do negócio são principalmente controlados por grupos multinacionais. Um estudo recente sobre a cadeia produtiva da soja, do plantio à agroindustrialização, revelou que 40\% do negócio da soja no Brasil são, de fato, brasileiros e destes, 13,3\% são concentrados na terra, e 14,3\% na mão de obra (MEDINA; RIBEIRO; BRASIL, 2016). Apenas $12,4 \%$ da participação das empresas brasileiras estão nos setores mais intensivos em tecnologia e capital como produção de sementes $(2,4 \%)$, fertilizantes $(4,8 \%)$, defensivos $(0,6 \%)$, máquinas $(0,3 \%)$ e agroindústria $(4,4 \%)$ (MEDINA; RIBEIRO; BRASIL, 2016) (Figura 1). 
Figura 1 - Participação do capital brasileiro na cadeia produtiva da soja

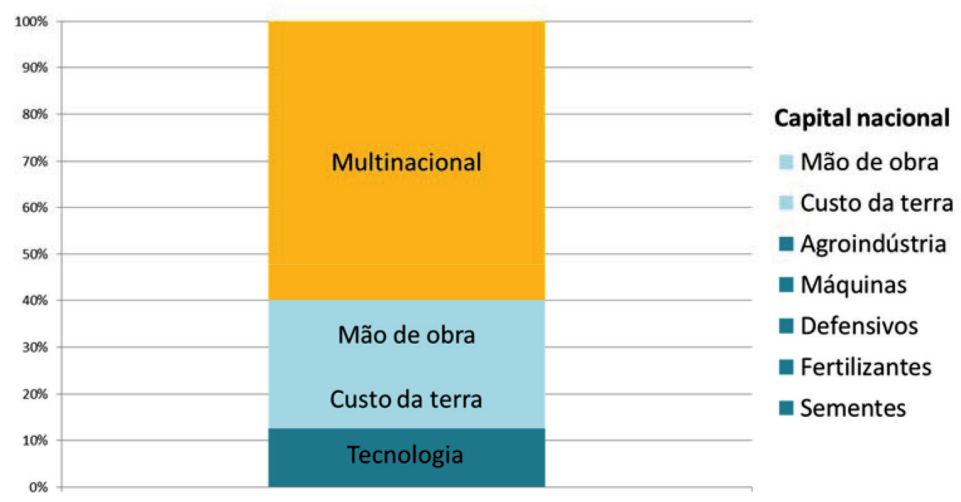

Fonte: Adaptado de Medina, Ribeiro e Brasil (2016)

Além da soja, estudos revelam que cadeias fundamentais do agronegócio brasileiro, como cana e frango, têm grande dependência tecnológica estrangeira (BASSI; SILVA; SANTOYO, 2013; MEURER et al., 2015). Essa dependência é particularmente relevante quando se considera que a tecnologia é responsável por $68 \%$ de incrementos na renda bruta dos produtores rurais (ALVES; ROCHA, 2010). A inovação feita no Brasil, adaptada às condições locais, pode aumentar a eficiência do setor e promover o desenvolvimento econômico nacional (ALTIERI; ROSSET, 1999; MALERBA; ORSENIGO, 1996).

Nesse sentido, a nova fase do desenvolvimento agrícola e agrário brasileiro tem pouco de brasileiro de fato que vá além de fatores de produção básicos como terra e mão de obra. O fato é relevante dada à importância da participação de grupos nacionais para o crescimento do país. $\mathrm{Na}$ história recente, economias em desenvolvimento diminuíram o atraso em relação às mais ricas na medida em que conseguiram alcançar níveis similares de conhecimento tecnológico e de qualificação da mão de obra, e não pela venda de ativos ao capital estrangeiro (PIKETY, 2014:75).

O futuro do agronegócio brasileiro passa pela construção de uma estratégia de integração vertical do capital nacional ao longo das cadeias produtivas. O avanço da produção agropecuária oferece espaços que podem ser mais bem ocupados por empresas nacionais. As oportunidades identificadas nas diferentes etapas da cadeia da soja incluem: 1. O fortalecimento de empresas nacionais produtoras de sementes; 2. A adoção de práticas alternativas de adubação com agentes biológicos e adubação mineral; 3. O manejo integrado de pragas como forma de reduzir custos de produção; 4. A participação no mercado de 
implementos agrícolas; 5. A consolidação de tradings nacionais atuando em cadeias regionais enfocadas no mercado interno(MEDINA; RIBEIRO; BRASIL, 2016). Há também exemplos de estratégias comercialmente mais agressivas a criação da gigante brasileira BRF(BASSI; SILVA; SANTOYO, 2013).

\section{A ESTRATÉGIA DE EXPANSÃO PARA NOVAS FRONTEIRAS AGRÍCOLAS, COM IMPACTOS SOCIAIS E AMBIENTAIS RELEVANTES, MANTÉM A QUESTÃO AGRÁRIA NA PAUTA}

Outra tese importante é que problemas históricos, como é o caso da “questão agrária” (BUAINAIN et al., 2013), perderam relevância em decorrência da expansão do agronegócio no Brasil. A antítese relacionada é que a estratégia atual de expansão do agronegócio para novas fronteiras agrícolas, com impactos sociais e ambientais relevantes, mantém a questão agrária na pauta (MEDINA; DOS SANTOS, 2017). O argumento é relevante, pois aponta para a necessidade de repensar a expansão desordenada da fronteira agrícola e de apoiar as populações locais com regularização fundiária (RAMOS, 2014). Adicionalmente, há indícios de que a demanda por reforma agrária tende a aumentar em épocas de crise e redução do emprego nos centros urbanos (FERNANDES, 2014).

Com a expansão das fronteiras agrícolas, surgem disputas pela posse da terra, muitas vezes gerando conflitos sociais (CPT, 2017). Esses conflitos acontecem mais comumente quando a população local, na condição de posseira, é confrontada com grandes produtores que migram em busca de novas terras (BENATTI, 2006). Há também casos semelhantes de grandes fazendeiros que invadem terras tradicionais indígenas ainda não demarcadas pelo governo (MASUZAKI, 2012). Dados da Comissão Pastoral da Terra (CPT) revelam que ocorrências de conflitos por terra em 2016 e 2017 são as mais elevadas desde quando a CPT começou a fazer este trabalho em 1985: 2016 - 1.079 ocorrências; 2017 - 989 ocorrências (CPT, 2017).

Há um crescimento recente no número de conflitos envolvendo povos indígenas. Um caso recente, emblemático do problema, foi o assassinato de membros da etnia Guarani-Kaiowá devido a conflitos relacionados à terra no estado de Mato Grosso do Sul. Entre 2003 e 2010, 452 assassinatos de indígenas foram registrados naquele estado, representando 55,5\% do total nacional (CIMI, 2011). Como tendência geral, há um aumento recente de conflitos onde a terra das populações tradicionais está em disputa com produtoresprovenientes de outras regiões (CPT, 2017). 
A expansão da fronteira agrícola também tem causado impactos ambientais relevantes (SILVA et al., 2010; SPERA et al., 2016)fertilizer, fuel, machines, pesticides and electricity. The expansion of this crop in Brazil in recent decades has generated concerns about its environmental impacts. To assess these impacts, two representative chains supplying soybeans to Europe were identified: Center West (CW, especialmente com o desmatamento de florestas nativas (FERREIRA et al., 2013; FERREIRA; COELHO, 2015). Estudos revelam que, em 2013, no bioma Cerrado, $13 \%$ da expansão total da soja ocorreram em detrimento da vegetação nativa (GIBBS et al., 2015). Na região da fronteira agrícola de Matopiba especificamente, cerca de $40 \%$ da expansão total de soja ocorreu em áreas de vegetação nativa (GIBBS et al., 2015). Em Matopiba, a expansão do agronegócio está ocorrendo nos estados com o maior percentual de vegetação nativa do bioma Cerrado: os estados do Maranhão, Tocantins, Piauí e Bahia que ainda têm, respectivamente, 89\%, 79\%, 91\% e 74\% de sua área total coberta por vegetação nativa (FERREIRA, 2016).

Na Amazônia, quase 30\% da expansão da soja até 2006 ocorreram em áreas de desmatamento, enquanto que em 2014 o desmatamento para o estabelecimento de áreas de soja caiu para cerca de 1\% do total (GIBBS et al., 2015). Tal redução é atribuídaà aplicação da legislação florestal pelo governo brasileiro (MACEDO et al., 2012), à moratória da soja, um acordo de desmatamento zero voluntário estabelecido em 2006(GIBBS et al., 2015), e à criação de territórios indígenas e unidades de conservação que protegem a vegetação nativa (NEPSTAD et al., 2014). Como as estratégias de expansão dosgrandes produtores emergem dos contextos em que estão inseridos (MEYFROIDT et al., 2014)for both global and domestic urban markets, follows multiple land change pathways entailing direct and indirect deforestation, and results in various social and environmental impacts. Here we compare six published case studies of rapid commodity crop expansion within forested tropical regions. Across cases, between 1.7\% and $89.5 \%$ of new commodity cropland was sourced from forestlands. Four main factors controlled pathways of commodity crop expansion: (i, uma questão ainda pouco explorada é como o controle da expansão da soja na Amazônia pode ter levado à maior expansão da cultura no Cerrado, caracterizando uma fuga (leakage) do desmatamento da Amazônia para o Cerrado.

O futuro do agronegócio brasileiro também passa pela superação da visão atual muitas vezes restrita à expansão horizontal para novas fronteiras agrícolas com altos impactos ambientais e sociais. É estratégico favorecer a consolidação das cadeias produtivas existentes com menores impactos e maiores benefícios para o país. 


\section{A APOSTA NA LIBERALIZAÇÃO DOS MERCADOS É FRUTO DE IGNORÂNCIA SOBRE AS POLÍTICAS AGRÍCOLAS DOS DEMAIS PAÍSES, QUE MANTÊM A PROTEÇÃO E OS SUBSÍDIOS A SEUS AGRICULTORES}

Outra tese estruturante alude à perda de relevância do Estado no apoio ao setor agrícola devido ao crescente protagonismo do segmento privado diante da liberalização dos mercados globais (BUAINAIN et al., 2013). A antítese relacionada mostra que a aposta na liberalização dos mercados agrícolas pode ser um equívoco diante da permanência das políticas agrícolas de outros países que mantiveram ou ampliaram a proteção e os subsídios a seus agricultores (MEDINA, 2018). O fato é relevante pois as dinâmicas internacionais têm implicações diretas no funcionamento dos mercados agrícolas e nas exportações agropecuárias brasileiras e precisam ser consideradas pela política nacional na construção da estratégia de desenvolvimento para o setor agropecuário.

O direcionamento da política agrícola brasileira mantém a aposta na liberalização dos mercados agrícolas internacionais como base de sustentação do desenvolvimento nacional via exportação de commodities agropecuárias (MORAES, 2014; SANTANA et al., 2014). Essa abordagem tem dominado a política brasileira desde a década de 1990 quando o país deu início ao processo de abertura comercial (SAES; SILVEIRA, 2014). Assim, a política agrícola do país tem ficado restrita ao atendimento das demandas mais imediatas apresentadas pelos produtores rurais e suas organizações representativas (CNA, 2018). A maior parte do crescente orçamento da política agrícola brasileira tem sido destinada ao crédito subsidiado para agricultores e pecuaristas, tanto no caso dos R 202 bilhões do Plano Agrícola e Pecuário 2017/18 voltado para grandes e médios produtores, quanto dos R \$ 30 bilhões do Plano Safra 2017/20 voltado para agricultores familiares (MAPA, 2017; SEAD, 2017).

Apesar da aposta brasileira na liberalização dos mercados agrícolas internacionais (SANTANA et al., 2014), mudanças recentes no cenário internacional devem ser consideradas pela política nacional. Estudos têm mostrado a manutenção das políticas agrícolas nos países desenvolvidos (MEDINA; POTTER; POKORNY, 2015), os crescentes investimentos feitos por países emergentes como China e Índia, assim como os limites para a liberalização dos mercados agrícolas enfrentados pela Rodada de Doha da Organização Mundial do Comércio (OMC)(OECD, 2015).

$\mathrm{Na}$ preparação para o Acordo sobre Agricultura (Agreement on Agriculture AoA), que foi implementado a partir de 1995, os estados-membros da OMC se 
comprometeram com a liberalização dos mercados agrícolas (POT'TER; TILZEY, 2007). As políticas nacionais tanto dos Estados Unidos quanto da Europa começaram a se afastar do uso de subsídios que distorcem mercado, como políticas de garantia de preços ao produtor, que foram classificadas como de uso restrito (POT'TER; TILZEY, 2007). Tanto Estados Unidos quanto Europa substituíram a política de preços por medidas que não distorcem o mercado, como o pagamento direto, que foi classificado como de uso irrestrito (HUIGE et al., 2010).

Agricultores de países desenvolvidos ligados à OECD ainda têm 17\% de sua renda garantida por transferências públicas (OECD, 2015). Na União Europeia esse valor é de 19\% e nos Estados Unidos, de 8\% (OECD, 2015), embora este último número suba para $37 \%$ se forem consideradas as transferências para o setor como um todo e não para os agricultores individualmente (EUROPEAN PARLIAMENT, 2012). Embora esses países tenham restringido o uso de subsídios que distorcem o mercado, seus investimentos em outros mecanismos de apoio têm sido crescentes (MEDINA; POT'TER; POKORNY, 2015) (Tabela 1).

Tabela 1 - Evolução do orçamento da PAC e Farm Bill e de seus principais instrumentos de apoio direto aos agricultores

\begin{tabular}{l|c|c|c|c}
\hline & $\begin{array}{c}\text { PAC } \\
\text { (Total)* }\end{array}$ & $\begin{array}{c}\text { PAC (Pagamento } \\
\text { direto)* }\end{array}$ & $\begin{array}{c}\text { Farm Bill } \\
\text { (Total)** }\end{array}$ & $\begin{array}{c}\text { Farm Bill } \\
\text { (Cropinsurance)** }\end{array}$ \\
\hline $\begin{array}{l}\text { Orçamento anual } \\
\text { médio para o período } \\
\text { anterior (2007-2013 } \\
\text { na PAC e 2008-2013 } \\
\text { na Farm Bill) (R\$ } \\
\text { bilhões) }\end{array}$ & 208,0 & 165,6 & 198,8 & 25,8 \\
\hline $\begin{array}{l}\text { Orçamento anual } \\
\text { médio para o período } \\
\text { atual (2014-2020 na }\end{array}$ & 228,0 & 168,0 & 342,0 & \\
$\begin{array}{l}\text { PAC e 2014-18 na } \\
\text { Farm Bill) (R bilhões) }\end{array}$ & & & & 29,1 \\
\hline Crescimento (\%) & 9,6 & 1,4 & 72,0 & 1,2 \\
\hline
\end{tabular}

Fonte: *European Parliament (2012); EUROPEAN COMISSION, 2013; ** JOHNSON; MONKE, 2014.

A Política Agrícola Comum da União Europeia (PAC) tem concentrado seus esforços na complementação de renda do produtor rural pelo pagamento direto. Dos cerca de 57 bilhões de euros ( $\mathrm{R} \$ 228$ bilhões) investidos anualmente na agricultura pela PAC, o pagamento direto é feito pelo primeiro pilar da política, que representa $74 \%$ do orçamento (COMISSION, 2013). O orçamento do pagamento direto tem estado estável ao longo dos últimos anos, com leve 
crescimento entre o período de 2007-2013 e 2014-2020 (MEDINA; POTTER, 2016). A Farm Bill americana está direcionada ao estabelecimento de uma rede de segurança (safety net) que protege o produtor em caso de perdas com a produção ou com os preços dos produtos agrícolas. Respectivamente $8,5 \%$ e 4,8\% dos 97,8 bilhões de dólares americanos ( $\mathrm{R} \$ 342$ bilhões) investidos anualmente pela Farm Bill vão para a proteção do produtor pelos programas Crop Insurance e Commodities Program (JOHNSON; MONKE, 2017). 80\% do orçamento vão para a compra de alimentos dos agricultores americanos pelo Estado (JOHNSON; MONKE, 2017).

A manutenção dos subsídios agrícolas a produtores americanos e europeus restringe as exportações brasileiras para esses países, que aumentaram graças ao crescimento da demanda chinesa, particularmente por soja(CONTINI, 2014). Não obstante, a China aumentou os investimentos em política agrícola para o equivalente a 3,2\% de seu PIB, e já subsidia cerca de $19 \%$ da renda do produtor rural chinês(OECD, 2015). A estratégia chinesa passa pela busca de maior autonomia na produção interna de cereais e pelo controle da estatal chinesa COFCO sobre cadeias de comercialização de produtos estratégicos, como soja, produzidos na América do Sul e África (GAUDREAU, 2015; ZHONG, 2009). A Índia, que tem a segurança alimentar de sua população como enfoque estratégico, decidiu reforçar sua política agrícola para subsidiar a produção nacional e proteger o mercado interno (KHORANA; PERDIKIS; KERR, 2015).

Nesse sentido, pode-se dizer que o investimento em políticas agrícolas é uma tendência internacional entre países desenvolvidos e em desenvolvimento a despeito dos esforços de liberalização dos mercados, dada a importância estratégica da agricultura para a segurança alimentar e para o desenvolvimento rural da maior parte dos países. Derivados da dificuldade de um acordo multilateral para a liberalização dos mercados agrícolas pelos 160 países que compõem a OMC, têm sido crescentes os esforços para acordos bilaterais e plurilaterais de comércio entre grupos restritos de países como é o caso da Parceria Transpacífico (TPP) e da Parceria Transatlântica de Comércio e Investimento (TTIP) (AKHTAR; JONES, 2013), embora o início do governo Donald Trump nos Estados Unidos sugira restrições ao TPP.

Em dezembro de 2013 um acordo sobre um pequeno subconjunto de questões foi alcançado no chamado Pacote de Bali, que é o primeiro acordo verdadeiramente multilateral negociado sob os auspícios da OMC (BELLMANN, 2014). A peça central do pacote é um novo acordo sobre a facilitação do comércio com o objetivo de reduzir a burocracia e facilitar os procedimentos 
aduaneiros em um esforço para reduzir o custo das exportações. No entanto, além da facilitação do mercado, os avanços sobre agricultura, desenvolvimento e países menos desenvolvidos são limitados (BELLMANN, 2014; PALIT, 2015). Um dos principais limites para o acordo em Bali foi colocado pelos países emergentes como a Índia. Pela necessidade de garantir a segurança alimentar da sua expressiva população rural, a Índia tem dificuldade de abrir mão da proteção da sua produção agrícola e das compras institucionais feitas pelo governo dos produtores rurais e de pequenos comerciantes locais (KHORANA et al., 2015).

As dinâmicas internacionais têm implicações diretas no funcionamento dos mercados e nas exportações agropecuárias brasileiras e precisam ser consideradas pela política agrícola nacional na construção da estratégia de desenvolvimento do setor agropecuário (JOSLING et al., 2015). O futuro do agronegócio brasileiro também passa pela atuação mais abrangente do Estado em frentes estratégicas como sistemas de informação, apoio institucional e infraestrutura, que podem trazer retornos para o setor rural, em superação ao enfoque atual quase exclusivo no atendimento aos interesses imediatistas dos produtores rurais por crédito agrícola subsidiado (MEDINA, 2018). Como exemplo, é estratégica a aposta em setores do agronegócio que exportam produtos com valor agregado a partir do uso de matéria-prima brasileira, como é o caso do mercado de carnes, que se vale da produção de ração à base de soja e milho (SANTANA et al., 2014).

\section{EXISTE GRANDE DIVERSIDADE ENTRE OS AGRICULTORES FAMILIARES NO BRASIL QUE PODEM SER ENCORAJADOS A PARTIR DE POLÍTICAS ESPECÍFICAS}

A última tese fundamental é a de que o Brasil viveria um desenvolvimento agrário bifronte,com pequena parcela de estabelecimentos altamente produtivos e a maior parte dos estabelecimentos, essencialmente de caráter familiar, com pouca relevância econômica e, portanto, redundante (BUAINAIN et al., 2013). A antítese relacionada é que existe grande diversidade de agricultores familiares no Brasil que podem ser encorajados a partir de políticas específicas (Quadro 1). $\mathrm{O}$ argumento é relevante pois indicaa viabilidade potencial de diferentes sistemas produtivos que dispõem de ativos como recursos naturais e força de trabalho e que poderiam ser apoiados com ações específicas.

De acordo com Alves e Rocha (2010), de todos os agricultores brasileiros, apenas 8,2\% têm renda acima de 10 salários mínimos por mês, 18,8\% entre duas e 10 vezes o salário mínimo e 73,0\% ganham menos de dois salários mínimos. 
$\mathrm{Na}$ última década, apenas 452.750 das explorações agrícolas familiares, que totalizam 4,3 milhões, conseguiram aumentar a participação na produção total, enquanto que o segmento mais pobre apenas cresceu em números absolutos, sem um aumento concomitante na produção (GUANZIROLI; BUAINAIN; SABBATO, 2013). Com esses fatos, existe certo consenso que apenas uma proporção limitada dos agricultores familiares brasileiros, particularmente aqueles da região Sul do país, teria as condições necessárias para se modernizar (MEDINA et al., 2015). A maior parte dos agricultores familiares no Brasil enfrenta constrangimentos estruturais em termos de recursos limitados, como parcelas de terra muito pequenas, ou contexto institucional desfavorável, como falta de acesso às políticas agrícolas, levando ao desenvolvimento limitado em termos de adoção tecnológica, de integração comercial e de organização social (MEDINA et al., 2015).

Apesar disso, pesquisas recentes revelam que mais do que uma agricultura "bifronte", o Brasil tem uma agricultura essencialmente heterogênea (GUANZIROLI; DI SABBATO, 2014). E as políticas precisam considerar a heterogeneidade entre os agricultores familiares, que contam com diferentes capacidades e vocações produtivas (MEDINA et al., 2015). A literatura sobre campesinato, embora amplamente negligenciada nos debates mais recentes, tem sido particularmente perspicaz em revelar características e potencialidades dos diferentes segmentos rurais brasileiros (WANDERLEY, 1999). Esses segmentos incluem não só os agricultores familiares modernos do Sul do Brasil, mas também os quilombolas (MARIN; CASTRO, 1998), os assentados (FERNANDES, 2014), os colonos (GODAR; TIZADO; POKORNY, 2012), os posseiros (GUERRA, 2001), os parceiros (CANDIDO, 1975), os grupos indígenas (POSEY, 1985) e as comunidades tradicionais (DIEGUES, 2000).

Estudiosos da área têm chamado atenção para um novo modelo de desenvolvimento rural baseado em canais curtos de comercialização, em agroindustrialização da produção e na pluriatividade (MALUF, 2004; SCHNEIDER, 2013; WILKINSON, 2003). O novo modelo de desenvolvimento rural representaria uma alternativa ao modelo de modernização da agricultura familiar pela integração aos canais convencionais de comercialização de commodities agrícolas. No entanto, ainda não existe uma avaliação quantitativa da abrangência desse novo modelo e de seu potencial para a inclusão social e produtiva da maior parte dos agricultores familiares brasileiros. 


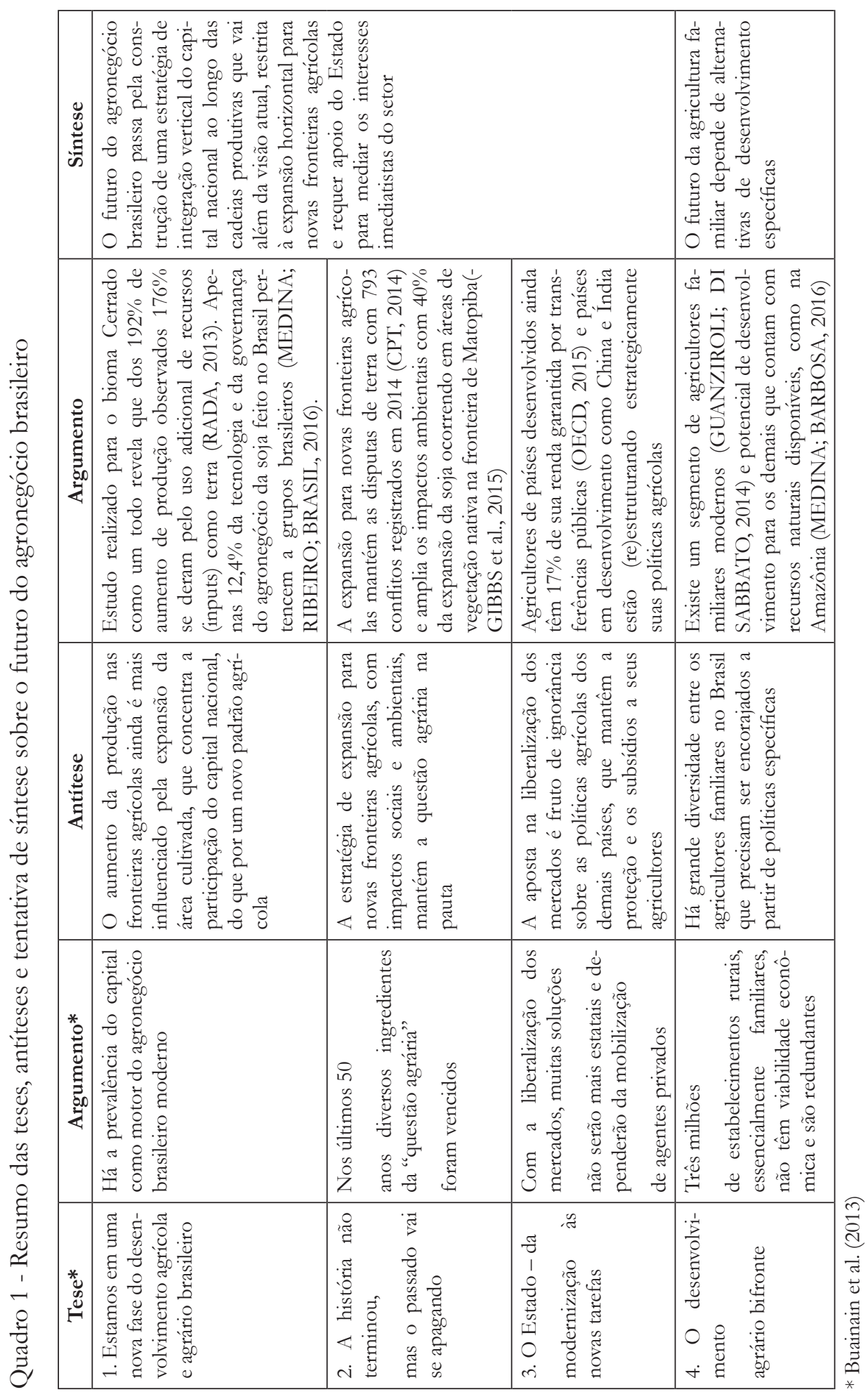


Para os casos em que os recursos naturais estão disponíveis e os agricultores sabem como gerenciar os ecossistemas locais é necessária uma alternativa à abordagem modernizadora que seja construída de acordo com as capacidades existentes (MEDINA; BARBOSA, 2015). Mesmo em territórios empobrecidos, estudos revelam possibilidades de desenvolvimento pela via da produção agropecuária para a maior parte dos agricultores familiares (MEDINA; NOVAES; TEIXEIRA, 2017). Na região amazônica, por exemplo, além da disponibilidade de recursos naturais, a população rural é majoritariamente jovem (GORI; BUAINAIN, 2015). E, mais importante, as comunidades locais têm sistemas tradicionais de gestão dos recursos naturais que podem formar a base para alternativas de desenvolvimento rural (MEDINA; BARBOSA, 2016). Portanto, é essencial que os sistemas de produção locais sejam reconhecidos e apoiados. O futuro da agricultura familiar depende de alternativas de desenvolvimento específicas para potenciais locais específicos, considerando a grande heterogeneidade do segmento.

\section{CONCLUSÃO}

As três primeiras antítesesapontam para o aumento da área cultivada como fator importante para o avanço do agronegócio feito no Brasil com pouca participação do capital brasileiro, para os impactos negativos da estratégia de expansão para novas fronteiras agrícolas e para a relevância de uma política agrícola estratégica e de longo prazo. Como esforço de síntese, essa situação revela que o futuro do agronegócio brasileiro passa pela construção de uma estratégia de integração vertical do capital nacional ao longo da cadeia produtiva, indo além da expansão horizontal para novas fronteiras agrícolas em que o Estado tem o papel fundamental de promover ações de longo prazo não restritas aos interesses imediatistas do setor.

A quarta antítese aponta para o fato de que os agricultores de menor escala produtiva, que são essencialmente agricultores familiares, representam uma grande diversidade possibilidades de desenvolvimento. Embora a atual política nacional de apoio à modernização do setor seja uma conquista importante, os resultados têm sido restritos a uma fração dos agricultores concentrados na região Sul. Como esforço de síntese, pode-se indicar que o futuro da agricultura familiar depende de alternativas de desenvolvimento específicas para atender a potenciais específicos. Um caso emblemático do potencial de desenvolvimento é a Amazônia, onde os ativos naturais estão disponíveis e os agricultores têm conhecimento sobre a gestão dos ecossistemas. 
Diante do cenário internacional dinâmico, a política agrícola brasileira precisa evoluir do atual enfoque quase exclusivo ao crédito subsidiado em resposta a pressões imediatistas dos produtores rurais organizados, para investimentos mais abrangentes podem trazer maiores retornos em longo prazo para o setor rural como um todo. Existe a necessidade de se considerar estrategicamente o mapeamento constante das dinâmicas do mercado internacional de produtos agropecuários e suas implicações para a agricultura nacional. $\mathrm{O}$ apoio a grupos nacionais estabelecidos ao longo das cadeias produtivas do agronegócio (dos produtores às tradings) é importante para aumentar a participação do capital brasileiro no agronegócio feito no Brasil, com maiores retornos financeiros para o país.

\section{REFERÊNCIAS}

AKHTAR, S.; JONES, V. Proposed Transatlantic Trade and Investment Partnership (TTIP): In Brief Congressional Research Service. [s.l: s.n.]. Disponível em: <https://www.fas.org/sgp/crs/row/R43158.pdf>. Acesso em: 10 de out. de 2016.

ALTIERI, M.; ROSSET, P. Ten reasons why biotecnology will not ensure food security, protect the environment and reduce poverty in the developing world. Ag Bio Forum, v. 2, 3, 4, p. 155-162, 1999.

ALVES, E.; ROCHA, D. D. P. Ganhar tempo é possível? In: GASQUES, J.; VIEIRA, A. (Ed.). A agricultura brasileira. Brasília: MDA, 2010. p. 275-290

BASSI, N. S.S.;SILVA, C.L.DA;SANTOYO, A. Inovação, pesquisa e desenvolvimento na agroindústria avícola brasileira. Estudos Sociedade e Agricultura, v. 21, n. 2, p. 392-417, 2013.

BELLMANN, C. The Bali Agreement: Implications for Development and the WTO.” International Development Policy, 5(2), 2014

BENATTI, J. A grilagem de terras públicas na Amazônia brasileira. Brasília: Ministério do Meio Ambiente, 2006.

BUAINAIN, A. et al. Sete teses sobre o mundo rural brasileiro. Revista de Política Agrícola, v. 22, n. 2, p. 1-25, 2013.

BUAINAIN, A. et al. O mundo rural no Brasil do século 21: A formação de um novo padrão agrário e agrícola. [s.l: s.n.]. 2014

CANDIDO, A. Os parceiros do Rio Bonito: estudo sobre o caipira paulista e a transformação dos seus meios de vida. São Paulo: Livraria Duas Cidades, 1975. 
CIMI. As violências contra os povos indígenas em Mato Grosso do Sul e as resistências do vem viver por uma terra sem males. Brasília: CIMI, 2011.

CNA. O Futuro é Agro 2018-2023. Brasília: [s.n.].

COMISSION, E. Overview of CAP Reform 2014-2020, 2013.

CONTINI, E. Exportações na dinâmica do agronegócio brasileiro: oportunidades econômicas e responsabilidade mundial. In: $\mathbf{O}$ mundo rural no Brasil do século 21: a formação de um novo padrão agrário e agrícola. [s.l: s.n.]. p. 147-174.

CPT. Conflitos no Campo - Brasil 2014. Goiânia: Comissão Pastoral da Terra, 2014.

CPT. Conflitos no campo no Brasil 2017. 1. ed. Goiânia: Comissão Pastoral da Terra, 2017.

DIEGUES, A. C. Os saberes tradicionais e a biodiversidade no Brasil. São Paulo: Ministério do Meio Ambiente, 2000.

EUROPEAN COMISSION, E. Overview of CAP Reform 2014-2020. [s.l: s.n.].

EUROPEAN PARLIAMENT. Latest U.S. Farm Bill Developments. [s.l: s.n.].

FERNANDES, B. M. Conflictuality, agrarian reform and development. Revista de Geografia Agrária, p. 1-10, 2014.

FERREIRA, M. et al. Considerations about the land use and conversion trends in the savanna environments of Central Brazil under a geomorphological perspective. Journal of Land Use Science, p. 1-15, 2013.

FERREIRA, M. Cerrado: O fim da história ou uma nova história? Ciência Hoje, v. 56, p. 334-340, 2016.

FERREIRA, M.; COELHO, A. Desmatamento Recente nos Estados da Amazônia Legal: uma análise da contribuição dos preços agrícolas e das políticas governamentais. Revista de Economia e Sociologia Rural, v. 53, n. 1, p. 91-108, 2015.

GASQUES, J. Produtividade total dos fatores e transformações da agricultura brasileira: análises dos dados dos censos agropecuários. In: GASQUES, J.; VIEIRA FILHO, J. E.; NAVARRO, Z. (Ed.). A agricultura brasileira: desempenho, desafios e perspectivas. Brasília: IPEA, 2010. p. 19-44.

GASQUES, J. et al. Produtividade da agricultura: resultados para o Brasil e estados selecionados. Revista de Política Agrícola, v. XXIII, n. 3, p. 87-98, 2014. 
GASQUES, J. G.; BACCHI, M. R. P.; BASTOS, E. T. Crescimento e Produtividade da Agricultura BrasileiraCarta de Conjuntura. Brasília: [s.n.]. Disponível em: <http://www.ipea.gov.br/cartadeconjuntura/index.php/2018/03/02/crescimentoe-produtividade-da-agricultura-brasileira-de-1975-a-2016/>.

GAUDREAU, M. Land grabbing, conflict and agrarian-environmental transformations: perspectives from East and Southeast Asia. Chiang Mai: [s.n.].

GIBBS, B. H. K. et al. Brazil's Soy Moratorium. Science, v. 347, n. 6220, p. 377-378, 2015.

GODAR, J.; TIZADO, E. J.; POKORNY, B. Who is responsible for deforestation in the Amazon? A spatially explicit analysis along the Transamazon Highway in Brazil. Forest Ecology and Management, v. 267, p. 58-73, mar. 2012.

GORI, A.; BUAINAIN, A. M. O novo mapa da população rural brasileiro. Confins, v. 25 , n. 25 , p. $1-26,2015$.

GRAZIANO DA SILVA, J. O Novo Rural Brasileiro. Nova Economia, p. 1-28, 2013.

GUANZIROLI, C.; BUAINAIN, A.; SABBATO, A. Family farming in Brazil: evolution between the 1996 and 2006 agricultural censuses. Journal of Peasant Studies, v. 40, n. 5, p. 817-843, 2013.

GUANZIROLI, C.; DI SABBATO, A. Existe na agricultura brasileira um setor que corresponde ao "Family Farming" Americano? Revista de Economia e Sociologia Rural, v. 52, p. 85-104, 2014.

GUERRA, G. A. D. O Posseiro da fronteira: campesinato e sindicalismo no sudeste paraense. Belém: Universidade Federal do Pará, 2001.

HUIGE, R.; LAPPERRE, R.; STANTON, G. The WTO Context. Wageningen: Wageningen University, 2010

JOHNSON, R.; MONKE, J. What Is the Farm Bill? Congressional Research Service. [s.l: s.n.].

JOSLING, T. et al. Latin American agriculture in a world of trade agreements. American Journal of Agricultural Economics, v. 97, n. 2, p. 546-567, 2015.

KHORANA, S.; PERDIKIS, N.; KERR, W. A. Global economies of scale in the EU-India trade agreement: are they the key to a return to economic growth? Asia Europe Journal, v. 13, n. 1, p. 41-55, 2015.

MACEDO, M. N. et al. Decoupling of deforestation and soy production in the southern Amazon during the late 2000s. Proceedings of the National Academy of Sciences, v. 109, n. 4, p. 1341-1346, 2012. 
MALERBA, F.; ORSENIGO, L. Schumpeterian patterns of innovation are technology-specific. Research Policy, v. 25, n. 3, p. 451-478, 1996.

MALUF, R. S. Mercados agroalimentares e a agricultura familiar no Brasil : agregação de valor, cadeias integradas e circuitos regionais Palavras-chave. Ensaios FEE, v. 25, n. 1, p. 299-322, 2004.

MAPA. Plano agrícola e pecuário 2017/2018. Brasília. Ministério da Agricultura, Pecuária e Abastecimento, , 2017.

MAPA, M. DA A. P. E A. Plano agrícola e pecuário 2016/2017. [s.l: s.n.].

MARIN, R. A.; CASTRO, E. Negros do Trombetas: guardiães de Matas e Rios. 2. ed. Belém: CEJUP, 1998.

MASUZAKI, T. I. A luta dos povos Guarani no extremo oeste do Paraná. Mundo do Trabalho, v. 16, p. 75-88, 2012.

MDA, M. DO D. A. Plano Safra da Agricultura Familiar 2016-2017. [s.l: s.n.].

MEDINA, G. et al. Development Conditions for Family Farming: Lessons From Brazil. World Development, v. 74, p. 386-396, 2015.

MEDINA, G. Agropecuária brasileira diante das dinâmicas internacionais. 1. ed. Goiânia: Editora UFG, 2018. v. 39

MEDINA, G.; BARBOSA, C. Experiências produtivas de agricultores familiares da Amazônia. Goiânia: Kelps, 2015.

MEDINA, G.; BARBOSA, C. A questão produtiva nas Reservas Extrativistas. Novos Cadernos NAEA, v. 19, n. 2, p. 69-88, 2016.

MEDINA, G.; DOS SANTOS, A. Curbing enthusiasm for Brazilian agribusiness: The use of actor-specific assessments to transform sustainable development on the ground. Applied Geography, v. 85, p. 101-112, 2017.

MEDINA, G.; NOVAES, E.; TEIXEIRA, S. M. Desenvolvimento local em territórios empobrecidos: possibilidades de inclusão social e produtiva de produtores rurais. Interações (Campo Grande), v. 18, n. 1, 10 mar. 2017.

MEDINA, G.; POT'TER, C. The nature and developments of the Common Agricultural Policy: Lessons for European Integration from the UK perspective. Journal of European Integration, v. In press, 2016.

MEDINA, G.; POTTER, C.; POKORNY, B. Farm business pathways under agri-environmental policies: Lessons for policy design. Estudos Sociedade e Agricultura, v. 23, n. 1, p. 5-30, 2015.

MEDINA, G.; RIBEIRO, G.; BRASIL, E. Participação brasileira na cadeia da soja: lições para o futuro do agronegócio nacional. Revista de Economia e Agronegócio, v. 16, n. 1, 2016. 
EDINA, G.; SANTOS, A. Dynamics of the Brazilian Countryside: An ActorSpecific Assessment on Possibilities for Sustainable Agricultural Development. Development and Change, v. In press, 2016.

MEURER, P. A. S. et al. Análise da Agroindústria Canavieira nos Estados do Centro-Oeste do Brasil a partir da Matriz de Capacidades Tecnológicas. Revista de Economia e Sociologia Rural, v. 53, n. 1, p. 159-178, 2015.

MEYFROIDT, P. et al. Multiple pathways of commodity crop expansion in tropical forest landscapes. Environmental Research Letters, v. 9, n. 7, p. $74012,2014$.

MORAES, L. A. M. DE. Brazil's agricultural policy developments. Revista de Política Agrícola, v. 1, n. 2, p. 55-64, 2014.

NEPSTAD, D. et al. Slowing Amazon deforestation through public policy and interventions in beef and soy supply chains. Science, v. 344, n. 6188, p. 1118-23, 2014.

OECD. Agricultural Policy Monitoring and Evaluation 2015. [s.l: s.n.].

OLIVEIRA, D.; SCHNEIDER, S. A. O futuro das unidades familiares: uma análise das possibilidades de sucessão hereditária entre os agricultores ecologistas de Ipê (RS). Novos Cadernos NAEA. v.12, n. 2, p. 149-174, dez. 2009

PEROBELLI, F. et al. Avaliação espacial das fontes de crescimento de um conjunto de commodities agrícolas brasileiras exportáveis entre 2003-2013. Revista de Economia e Agronegócio, v. 14, n. 1,2 e 3, p. 3-40, 2016.

PALIT, A. Mega-RTAs and LDCs: Trade Is Not for the Poor. Geoforum, 58:23-26, 2015.

PIKETY, T. O capital no século XXI. Rio de Janeiro: Intrínseca, 2014.

POSEY, D. Indigenous management of tropical forest ecosystems: the case of the Kayapo indians of the Brazilian Amazon. Agroforestry Systems, v. 3, n. 2, p. 139158, 1985.

POTTER, C.; TILZEY, M. Agricultural Multifunctionality, Environmental Sustainability and the WTO: Resistance or Accommodation to the Neoliberal Project for Agriculture? Geoforum, 38(6):1290-1303, 2007.

RADA, N. Assessing Brazil's Cerrado agricultural miracle: An update. Food Policy, v. 38, p. 146-155, 2013.

RAMOS, P. Uma história sem fim: a persistência da questão agrária no Brasil contemporâneo. In: $\mathbf{O}$ mundo rural no Brasil do século 21: a formação de um novo padrão agrário e agrícola. [s.l: s.n.]. 2014. p. 655-694. 
SAES, M. M.; SILVEIRA, R. L. F. Agropecuárias Brasileiras : Tendências Recentes. Estudos Sociedade e Agricultura, v. 22, n. 2, p. 386-07, 2014.

SAES, M. M.; SILVEIRA, R. L. F. Novas formas de organização nas cadeias agropecuárias brasileiras: Tendências recentes. Estudos Sociedade e Agricultura, v. 22, n. 2, p. 386-407, 2014.

SANTANA, C. et al. Política agrícola: avanços e retrocessos ao longo de uma trajetória positiva. In: $\mathbf{O}$ mundo rural no Brasil do século 21: a formação de um novo padrão agrário e agrícola. [s.l: s.n.]. p. 795-826.

SCHNEIDER, S. Situando o desenvolvimento rural no Brasil: o contexto e as questões em debate. Revista de Economia Política, v. 30, n. 3, p. 511-531, 2010.

SCHNEIDER, S. A pluriatividade como estratégia de reprodução social da agricultura familiar no Sul do Brasil. Estudos Sociedade e Agricultura, v. 16, p. 164-184, 2013.

SEAD. Plano Safra da Agricultura Familiar: 2017-2020: Fortalecer o campo para desenvolver o Brasil. Brasília. Secretaria Especial de Agricultura Familiar e do Desenvolvimento Agrário, , 2017. Disponível em: <http://www.mda.gov.br/ sitemda/sites/sitemda/files/user_img_1684/3Baixa_Cartilha_Plano_Safra_2017. pdf $>$

SILVA, P. et al. Variability in environmental impacts of Brazilian soybean according to crop production and transport scenarios. Journal of Environmental Management, v. 91, n. 9, p. 1831-1839, 2010.

SPERA, S. et al. Land-Use Change Affects Water Recycling in Brazil's Last Agricultural Frontier. Global Change Biology, v. 1, n. 1, p. 1-34, 2016.

VALOR. Cofco compra 49\% restantes da Noble Agri por US\$ 750 milhões. Valor, p. http://www.valor.com.br/agro/4368142/cofco-compra-, 2015.

VEIGA, J. O Brasil rural precisa de uma estratégia de desenvolvimento. Brasília: [s.n.]. v.1, 2001.

WANDERLEY, M. Raízes históricas do campesinato brasileiro. Agricultura familiar: realidades e perspectivas, v. 3, p. 21-55, 1999.

WILKINSON, J. A agricultura familiar ante o novo padrão de competitividade do sistema agroalimentar na América Latina. Estudos Sociedade e Agricultura, n. October, 2003.

ZHONG, F. China's agricultural policy. In: KAOSA-ARD, M.; ADAM, A. (Eds.). . Feeding the dragon: agriculture - China and the GMS. 1. ed. Chiang Mai: Chiang Mai University, 2009. p. 280. 